\title{
Application of Artificial Sunlight for the Elderly as a Possible Environmental Nursing Practice
}

\author{
Shigeru Goto ${ }^{1,3,4,5^{*}}$, Toshiaki Nakano, ${ }^{2,3}$, Chao-Long Chen ${ }^{3}$, King-Wah Chiư ${ }^{3}$, Li-Wen Hsu ${ }^{3}$, Seiko I ${ }^{4}$ I-Hsuan Chen ${ }^{3}$, \\ Kuang-Tzu Huang ${ }^{3}$, Ding-Wei Chen ${ }^{3}$, Takeshi Goto ${ }^{1}$, Naoya Omori ${ }^{1}$, Syuji Sato ${ }^{1}$, Hideki Kai ${ }^{4}$, Wataru Tsuruta ${ }^{4}$, \\ Masahiko Kai ${ }^{4}$, Simon Peter Bahau ${ }^{6}$, Yuki Takaoka ${ }^{7}$, Eriko Tane ${ }^{1}$, Kayoko Yuyama ${ }^{1}$, Chieko Wano ${ }^{1}$, Eiko Inoue ${ }^{1}$, \\ Roger Lord ${ }^{8}$ and Kanae lida ${ }^{1}$
}

\author{
${ }^{1}$ Faculty of Nursing, Department of Nursing, Josai International University, Chiba, Japan \\ ${ }^{2}$ Graduate Institute of Clinical Medical Sciences, Chang Gung University College of Medicine, Kaohsiung, Taiwan \\ ${ }^{3}$ Liver Transplantation Center, Kaohsiung Chang Gung Memorial Hospital, Kaohsiung, Taiwan \\ ${ }^{4}$ Fukuoka Institute of Occupational Health, Fukuoka, Japan \\ ${ }^{5}$ Mibyou Medical Institute AKOU, Yufuin, Oita, Japan \\ ${ }^{6}$ Center for International Education and Research, Toyama University, Toyama, Japan \\ ${ }^{7} Q$-may Laboratory Corporation, Oita, Japan \\ ${ }^{8}$ School of Science, Faculty of Health Sciences, Australian Catholic University, Brisbane, Australia
}

\begin{abstract}
*Corresponding Author: Shigeru Goto, M.D.,Ph.D, Professor, Faculty of Nursing, Department of Nursing, Josai International University, Chiba, Japan. 1 Gumyo, Togane, Chiba, 283-8555, Japan, Tel: + 81-475-55-7539; Fax: +81-475-55-8811; Email: sgoto@jiu.ac.jp
\end{abstract}

Received Date: December 22, 2017 Accepted Date: February 01, 2018 Published Date: February 08, 2018

Citation: Shigeru Goto, Toshiaki Nakano, Chao-Long Chen, King-Wah Chiu, Li-Wen Hsu, Seiko I, et al. (2018). Application of Artificial Sunlight for the Elderly as a Possible Environmental Nursing Practice.

POJ Nurs Prac Res 2 (1): 1-5.

\begin{abstract}
Aging and aged societies have arrived in many countries where significant development of medicine and the economy has been achieved. Japan is a highly aged society with a shortage of carers both at home and in long-term care settings. In fact, more persons of advanced age who do not need intensive care in hospitals strongly desire health care to be delivered in their own home with their family. Environmental nursing practice, which means that nurses improve the environment of patients or the elderly properly in accordance with healthcare considerations, is currently playing a more important role, not only to prevent the elderly from contracting various diseases, but also to provide a facility for supporting an aged person to live a fulfilling life, preferably one that includes independence. This approach will lead to a reduction in medical expenditure by increasing the number of aged people with healthy longevity without the need for hospitalization or intensive care. This short communication focuses on healthy lighting for the elderly based on our research and experience regarding the beneficial effects of artificial sunlight on nonalcoholic steatohepatitis (NASH), asthma and food allergy, and ulcerative colitis in experimental animal models and clinical settings. Then, we review other studies and discuss how artificial sunlight would be useful for the elderly as one of the environmental nursing practices.
\end{abstract}

Keywords: Artificial Sunlight, Phototherapy, Elderly People, Environmental Nursing, Vitamin D

\section{Introduction}

In 1860, Florence Nightingale published "Notes on Nursing" and mentioned the importance of environmental factors including lighting and sunshine in securing the health of houses and hospitals [1]. As Nightingale described, considering and providing five essential points (pure air, pure water, efficient drainage, cleanliness and light) may be one of environmental nursing practice. Recently, many hospitals are starting to use light therapy to treat brain disorders by resetting the body clock [2]. In not only hospitals but also in houses, there are several health

Copyright:@ 2018 Shigeru Goto, et al. This is an open-access article distributed under the terms of the Creative Commons Attribution License, which permits unrestricted use, distribution, and reproduction in any medium, provided the original author and source are credited. 
advantages of ensuring that the level and exposure of adequate lighting are available in living spaces that house the elderly to maintain health and promote mortality. Implementation of such practices not only improves quality of life for the individual but also a considerable reduction costs associated with medical treatments and burden on society. In this short communication we consider a number of age related health concerns which can be better controlled by the application of artificial sunlight.

\section{Artificial sunlight for vitamin D3 deficiency and to treat fatty} liver disease in the elderly

Non-alcoholic fatty liver disease (NAFLD) affects mainly the middle-aged and the elderly. With advancing age come more risk factors for its development. Older patients show more severe biochemical, hematological and histological changes, with cirrhosis being associated with greater age than those with milder disease. Obesity and hepatic steatosis (alcoholic and NAFLD) are becoming increasingly common medical problems in populations of the developed world. Additionally, NAFLD includes both simple fatty liver and nonalcoholic steatohepatitis (NASH), defined by the presence of lobular necroinflammatory activity with or without the presence of perisinusoidal fibrosis on liver biopsy [3]. NASH is benign and principally a disease of middle and old age. However, recent studies suggest an increased mortality in the $>60$-year-olds with NASH [4]. NASH is recognized as the most severe form of NAFLD, with likely progression to liver cirrhosis and hepatocellular carcinoma. To date, the only reliable method of differentiating simple steatosis from NASH is by liver biopsy, which is costly and carries some invasive risk to patients. Furthermore, there is no unified standard for therapeutics. In our previous study, artificial sunlight (color temperature $5500 \mathrm{~K}$, color rendition index (CRI)>90Ra; Chang Gung Biotechnology, Taipei, Taiwan) (Figure 1 B) have been used for NASH models including LEW rats fed with cholinedeficient and iron-supplemented L-amino acid-defined (CDAA) diet and leptin receptor-mutant Zucker rats [5]. Briefly, this was achieved via phototherapy ameliorated hepatocyte apoptosis, inflammation, fibrosis and insulin/leptin resistance caused by a CDAA diet with alteration of the levels of lipid transfer/ metabolic proteins and elevation of the circulating active form

Figure 1: Spectrum of artificial sunlight

A. Normal light

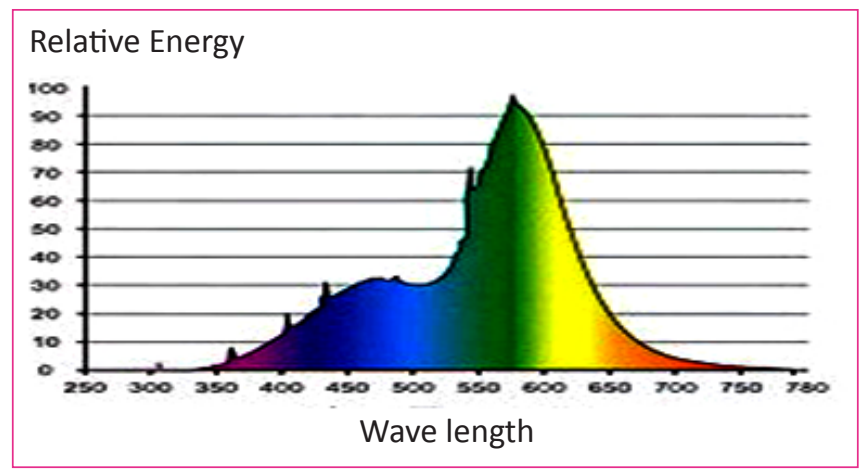

B. Artificial sunlight (color temperature $5500 \mathrm{~K}$, color rendition index (CRI)>90Ra; Chang Gung Biotechnology, Taipei, Taiwan)
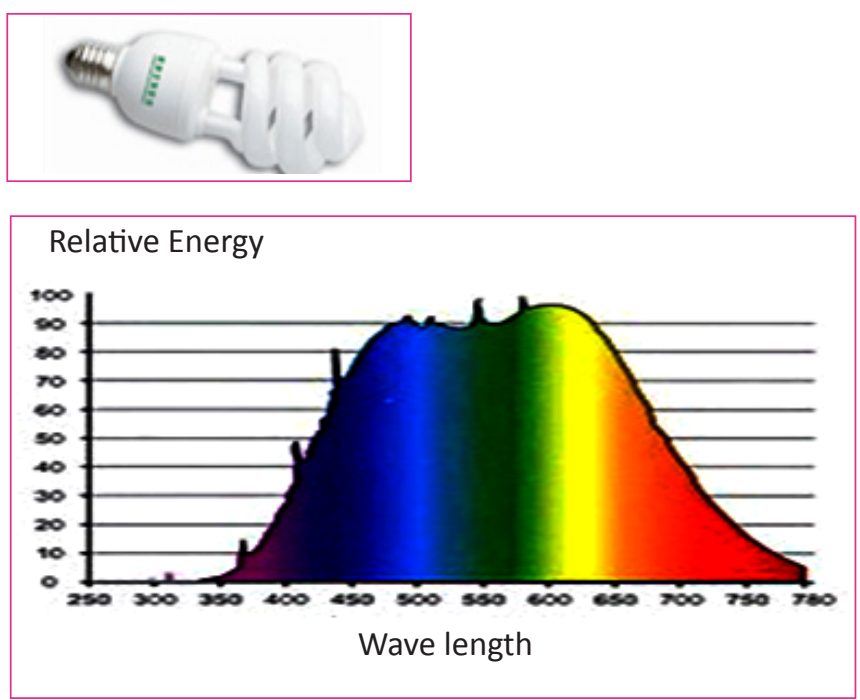

\section{Natural sunshine}

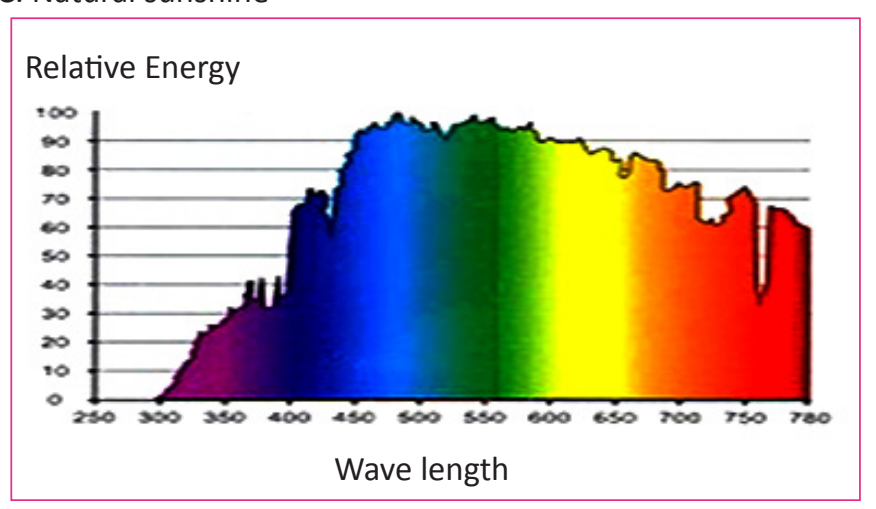

Figure 2: Serum level of 1 alpha,25-dihydroxyvitamin D3 before phototherapy and after 1 and 2 months following phototherapy for three NASH patients (65-70 year female)

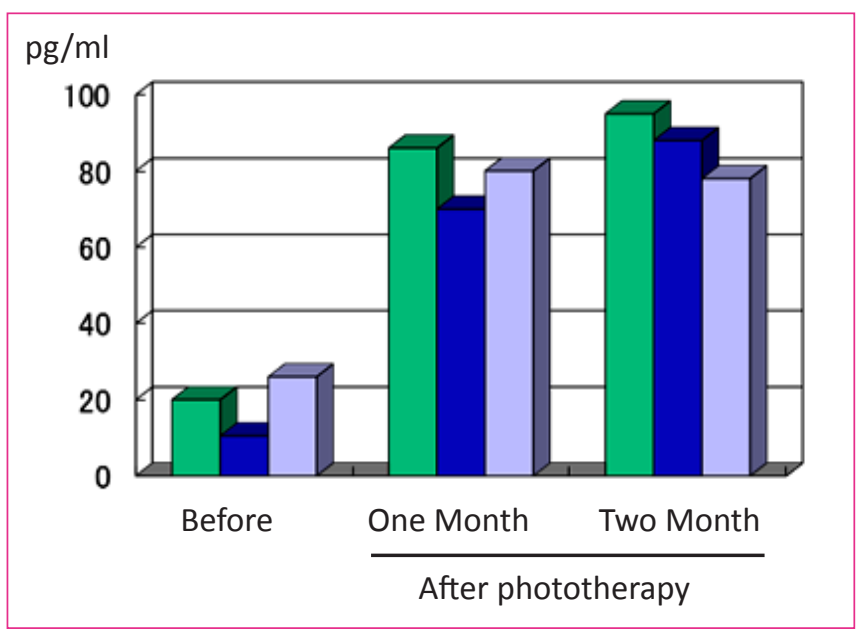

of vitamin D3. In addition, we have demonstrated that vitamin D3 (1 $\alpha$-hydroxy-cholecalciferol, $1 \alpha(\mathrm{OH}) \mathrm{D} 3$, Teijin Pharma Limited, Tokyo, Japan) supplementation ameliorated NASH progression in the CDAA diet-induced NASH model $[5,6]$. 
This study was the first confirmation of the therapeutic potency of sunlight therapy and Vitamin D3 supplementation in an animal model of fatty liver disease, which clearly builds the basis for subsequent human therapeutic trials in NAFLD.

In clinical settings, liver function of three NASH patients improved in association with elevated level of activated vitamin D3 after they had undergone artificial sunlight therapy using heated carbon (Figure 4) for 15 minutes twice a week for three months (Figure 2). In living donor liver transplantation (LDLT), NASH is contra-indication as liver donors with NASH die after hepatectomy [7]. Phototherapy will not only alleviate the problem of limited organ availability by allowing the use of NASH donors for liver transplantation but also permit the exploration of possibly establishing non-invasive prophylaxis and therapy for contemporary medical problems such as metabolic diseases in the elderly.

\section{The effect of artificial sunlight on asthma and food allergy}

Asthma is a common disease found in people over age of 65 [8]. Asthma in older adults can cause serious health problems if not treated properly. Asthma creates a much greater risk for older adults because they are more likely to develop respiratory failure as a result of the asthma, even during mild episodes of symptoms. Additionally, the care giver for an older person must have careful consideration of environmental factors (dust, light, air, food and water, etc.) to avoid the induction of asthma. Excessive sunbathing is a cause of melanoma while appropriate sunbathing reportedly has an anti-inflammatory ability to facilitate excretion of inflammatory substances or an ability to promote repair and regeneration of damaged tissues $[9,10]$. However, the amount of time senior people spend sunbathing is decreasing due to changes in lifestyle or working commitments of carers for the elderly as well as their anxiety regarding the risk for sunburn or skin cancer caused by ultraviolet rays. Our experimental results clearly demonstrated that irradiation with artificial light appeared to have a beneficial effect on asthma through suppressive effects of associated serum IgE antibody and inflammatory cytokines in a mouse model of asthma [11].

Food allergy is common in children and the prevalence of food allergy in senior people has recently been shown to have also increased without exactly knowing why [12]. We recently confirmed that 12 hour exposure by non-harmful artificial sunlight could completely reduce the symptoms of egg allergy in conjunction with the down regulation of IgE in a mouse food allergy model (manuscript in preparation).

\section{Artificial sunlight therapy for inflammatory bowel diseases}

The incidence and prevalence of inflammatory bowel diseases (IBD) such as Crohn's disease (CD) and ulcerative colitis (UC) is increasing not only in the young generation but also in the elderly [13]. The clinical features and therapeutic options in young and elderly patients are comparable but there is no reliable guideline for the diagnosis and treatment of IBD in elderly patients. Medical treatment for IBD requires chronic administration of anti-inflammatory drugs including corticosteroids, aminosalicylates, immunosuppressant drugs and antibiotics which often cause significant side effects. We have demonstrated that non-invasive artificial sunlight phototherapy improves dextran sulfate sodium (DSS)-induced colitis in a mouse model of IBD by suppression of pro-inflammatory (Th1) cytokines and promotion of anti-inflammatory (Th2) cytokines [14].

\section{Brief review of other sunlight-related studies}

Accumulated evidence has demonstrated the effect and association of natural and artificial sunlight with various diseases. It has been reported that insufficient day light exposure affects peripheral clock gene expression, suppresses corticosterone secretion and inhibits melatonin secretion, locomotor activity, body temperature and heart rate. We also have partially demonstrated the beneficial effects of artificial sunlight on $\mathrm{NASH}$, allergy, osteoporosis, carcinogenesis, insomnia and depression can be influenced by the elevation of activated vitamin D3 and regulation of time clock genes following sunlight. A broad consensus has been reached that vitamin $D$ deficiency is associated with increased risks of deadly cancers, cardiovascular disease, osteoporosis, multiple sclerosis, rheumatoid arthritis, and type 1 diabetes mellitus although chronic excessive exposure to sunlight increases the risk of melanoma and skin cancer.

The avoidance of all direct sun exposure increases the risk of vitamin D deficiency, which can have serious consequences. A lower level of activated vitamin D3 by avoiding sun exposure is a risk factor for all-cause mortality [15]. Avoidance of sunshine exposure and the subsequent low level of activated vitamin D leads to osteoporosis in the elderly. Bone fracture often occurs in the elderly with osteoporosis upon falling and a major cause of mortality in the elderly. Monitoring the active derivative 1 alpha, 25-dihydroxyvitamin D3 in serum annually should help reveal vitamin $D$ deficiencies. Sensible sun exposure (usually 5-10 min of exposure of the arms and legs or the hands, arms, and face, 2 or 3 times per week) and increased dietary and supplemental vitamin $\mathrm{D}$ intakes are reasonable approaches to guarantee vitamin D sufficiency.

Vitamin D is recognized as the "sunshine vitamin." Most humans depend on sun exposure to satisfy their requirements for vitamin $D$, but it can be obtained from fortified food, oily fish and vitamin D supplements [16]. Solar ultraviolet B photons are absorbed by 7-dehydrocholesterol (provitamin D3, 7-DHC) in the skin, leading to its transformation to pre-vitamin D3, which is rapidly converted to vitamin D3. Once formed, vitamin D3 is metabolized in the liver to 25-hydroxyvitamin D3 $(25(\mathrm{OH})$ D3; calcidiol) and then in the kidney to its biologically active form, 1 alpha, 25-dihydroxyvitamin D3 (1,25(OH)2D3; calcitriol) [17]. By binding to vitamin D-binding protein (VDBP), a carrier protein in the plasma, calcitriol is transported to various target organs [18]. In addition to its well-known effects on the skeleton, vitamin $D$ deficiency has been recognized to lead to direct adverse effects on other organ systems. Accumulating research suggests that low calcidiol concentrations may be related to 
increased prevalence of various cancers, autoimmune diseases and cardiovascular events [18].

In our previous study, we conformed the induction of vitamin D3 by phototherapy reduced the side effects (e.g., pain, nausea, poor appetite) during chemotherapy in a patient with small bowel adenocarcinoma [19]. Additionally, circadian rhythms in the elderly often become reversed with quality of sleep being affected as a result. It has been reported that allocating living places in an institution's architectural setting and providing social opportunities for elderly persons to get direct sunlight exposure can help increase the sleep quality of older adults [20]. However, shortage of manpower of care takers especially for bed-ridden elderly makes even short-term daily sun exposure difficult.

\section{Clinical trials and future prospective studies}

The Liver Transplantation Center within the Kaohsiung Chang Gung Memorial Hospital, Taiwan has already set up one post-transplant intensive care ward whose ceiling lights were replaced from normal light to artificial sun lights (color temperature $5500 \mathrm{~K}$, color rendition index (CRI)>90Ra; Chang Gung Biotechnology, Taipei, Taiwan) (Figure 3). In this ward, we have already experienced that one post-liver transplant patient with severe jaundice dramatically recovered in association with an elevated serum level of Apo-E.

We have also seen that liver function of three NASH patients became normal in association with an elevated serum level of activated vitamin D3 when we treated them for $15 \mathrm{~min}$ twice a week with artificial sunlight using heated carbons (14800BZZ00510, Kokentou. Co.Ltd. Tokyo, Japan) (Figure 4). To elucidate more precisely the scientific mechanisms involved with phototherapy, cell based experiments or various disease animal models are currently being carried out under the different color of LED with different wavelengths. Many types of artificial sunlight are commercially available (Figure 5). However, in near future when LED phototherapy is well established with solid evidence by us and others, "custom-made" LED (different colors) phototherapy will be undertaken in the elderly who have different medical conditions to tailor treatment therapy to a specific condition.

At the time of writing, the Department of Nursing at Josai International University, Japan, is in charge of several prospective studies to evaluate the effect of artificial sunlight on individuals aged over 70 years 1) who are completely independent in daily living activities, or 2) who are diagnosed with fatty livers, osteoporosis, asthma, major depression, or having bad sleep quality using sleeping pills, in cooperation with nursing and caring facilities. Environmental nursing care for the elderly must also be tailor-made and different types of care needed for agedpeople with different illness trajectories [21]. Artificial sunlight may be one of active or palliative care, however, this kind of environmental management must be described in any care plan with the elderly and their carers following better understanding of the medical illness suffered and their trajectories.

Figure 3: A ward equipped with artificial sunlight (color temperature $5500 \mathrm{~K}$, color rendition index (CRI)>90Ra; Chang Gung Biotechnology, Taipei, Taiwan) in Liver Transplantation Center, Kaohsiung Chang Gung Memorial Hospital, Kaohsiung, Taiwan

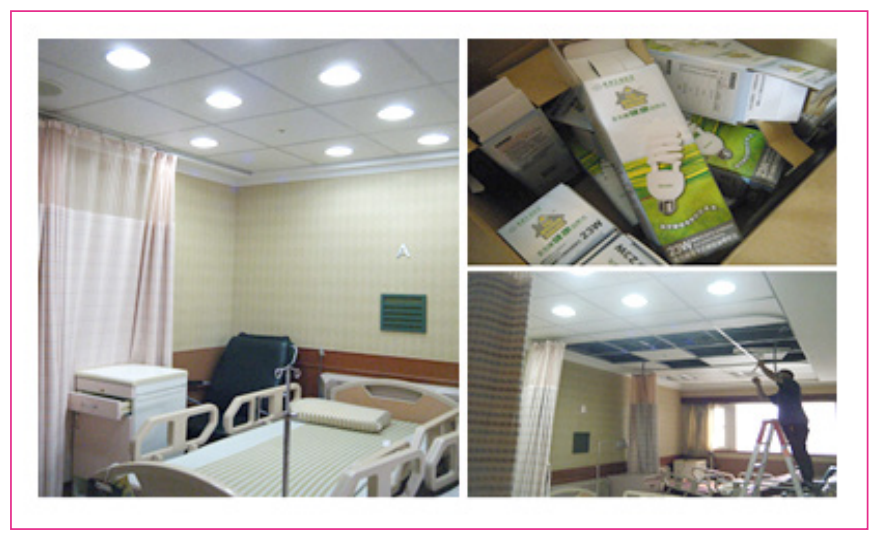

Figure 4: Artificial sunlight using heated carbons (14800BZZ00510, Kokentou. Co.Ltd. Tokyo, Japan)

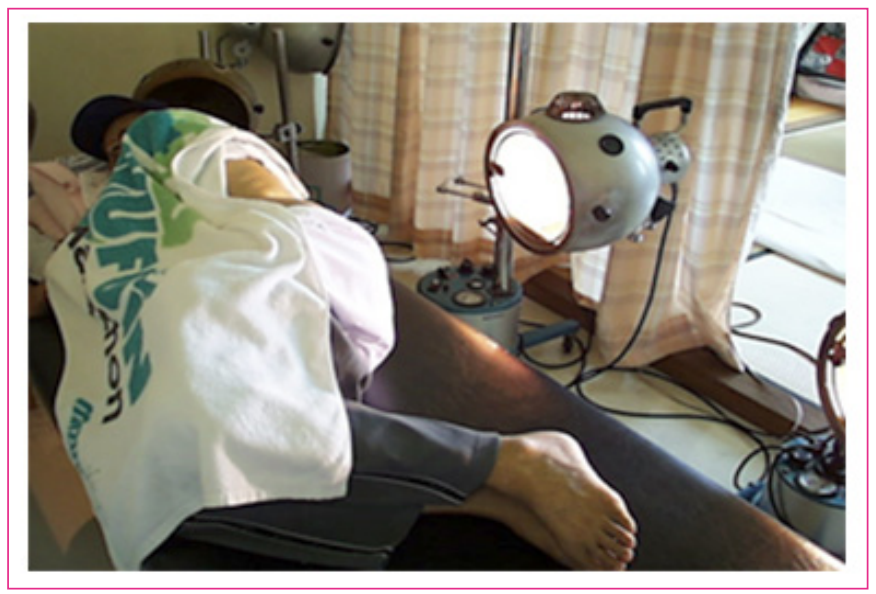

Figure 5: Various types of artificial sunlight

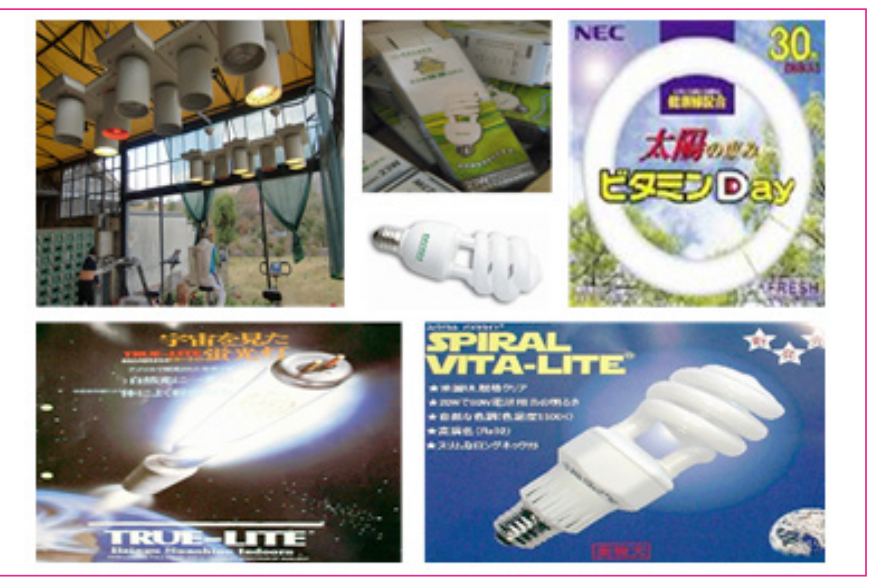

Declarations:

Conflict of Interest: The author declares no conflicts of interest.

Citation: Shigeru Goto, Toshiaki Nakano, Chao-Long Chen, King-Wah Chiu, Li-Wen Hsu, Seiko I, et al. (2018). Application of Artificial Sunlight for the Elderly as a Possible Environmental Nursing Practice. POJ Nurs Prac Res 2 (1): 1-5. 


\section{References}

1. Nightingale F. Notes on nursing; what it is, and what it is not. New York: D. Appleton and Company; 1860. [First American Edition]

2. Linda Geddes. Using light to reset the body clock can treat brain disorders. New Scientist, News \& Technology. [cited 2017 July 5]; Available from: https://www.newscientist. com/article/mg23531335-300-using-light-to-reset-thebody-clock-can-treat-brain-disorders/

3. Palekar NA, Naus R, Larson SP, Ward J, Harrison SA. Clinical model for distinguishing nonalcoholic steatohepatitis from simple steatosis in patients with nonalcoholic fatty liver disease. Liver Int. 2006;26(2):151-156.

4. Frith J, Day CP, Henderson E, Burt AD, Newton JL. Nonalcoholic fatty liver disease in older people. Gerontology. 2009;55(6):607-613.

5. Nakano T, Cheng YF, Lai CY, Hsu LW, Chang YC, Deng JY, et al. Impact of artificial sunlight therapy on the progress of non-alcoholic fatty liver disease in rats. J Hepatol. 2011;55(2):415-425.

6. Geier A. Shedding new light on vitamin D and fatty liver disease. J Hepatol. 2011;55(2):273-275.

7. Yamamoto K, Takada Y, Fujimoto $Y$, Haga H, Oike F, Kobayashi N, et al. Nonalcoholic steatohepatitis in donors for living donor liver transplantation. Transplantation. 2007;83(3):257-262.

8. Andrew Gillman, Jo A Douglass. Asthma in the elderly. Asia Pac Allergy. 2012;2(2):101-108.

9. Fears TR,Scotto J, Schneiderman MA. Skin cancer, melanoma, and sunlight. Am J Public Health. 976;66(5):461-4.

10. Brown SD, Calvert HH, Fitzpatrick AM. Vitamin D and asthma. Dermatoendocrinol. 2012;4(2):137-145.

11. Inomata $M$, Hiratsuka $T$, Seiko I, Goto S, Oyama $Y$, Nakano $\mathrm{T}$, et al. Therapeutic effects of artificial light therapy on allergic bronchial asthma in a mouse model. Adv Tech Biol Med. 2014;2(1):114.

12. Möhrenschlager $M$, Ring J. Food allergy: an increasing problem for the elderly. Gerontology. 2011;57(1):33-6.

13. Danielle Nimmons and Jimmy K Limdi. Elderly patients and inflammatory bowel disease. World J Gastrointest Pharmacol Ther. 2016;7(1):51-65.

14. Hiratsuka T, Inomata M, Goto S, Oyama $Y$, Nakano $T$, Chen $\mathrm{CL}$, et al. Phototherapy with artificial light suppresses dextran sulfate sodium-induced colitis in a mouse model. J Gastroenterol Hepatol. 2014;29(4):749-56.
15. Lindqvist $P G$, Epstein $E$, Landin-Olsson $M$, Ingvar $C$, Nielsen $\mathrm{K}$, Stenbeck $\mathrm{M}$, et al. Avoidance of sun exposure is a risk factor for all-cause mortality: results from the Melanoma in Southern Sweden cohort. J Intern Med. 2014;276(1):7786.

16. Grant WB, Holick MF. Benefits and requirements of vitamin D for optimal health: a review. Altern Med Rev. 2005;10(2):94-111.

17. Holick MF. Sunlight and vitamin D for bone health and prevention of autoimmune diseases, cancers, and cardiovascular disease. Am J Clin Nutr. 2004;80(6 Suppl):1678S-88S.

18. Holick MF. Vitamin D: importance in the prevention of cancers, type 1 diabetes, heart disease, and osteoporosis. Am J Clin Nutr. 2004;79(3):362-71.

19. Goto S, Iwao Y, Iwao J, Karahara K, Kitano S. A case report of a patient with small bowel adenocarcinoma undergoing combination therapies with chemotherapy and phototherapy. The Journal of The Japanese Society of Balneology, Climatology and Physical Medicine 2008;71(4):221-228.

20. Düzgün $G$, Durmaz Akyol A. Effect of natural sunlight on sleep problems and sleep quality of the elderly staying in the nursing home. Holist Nurs Pract. 2017;31(5):295-302.

21. Scott A Murray, Marilyn Kendall, Kristy Boyd, Azzi Sheikh. Illness trajectories and palliative care. BMJ. 2005;330(7498):1007-1011. 